\title{
Polaron effects and electric field dependence of the charge carrier mobility in conjugated polymers
}

\author{
Mattias Jakobsson and Sven Stafström
}

\section{Linköping University Post Print}

N.B.: When citing this work, cite the original article.

Original Publication:

Mattias Jakobsson and Sven Stafström, Polaron effects and electric field dependence of the charge carrier mobility in conjugated polymers, 2011, Journal of Chemical Physics, (135), 13, 134902.

http://dx.doi.org/10.1063/1.3644931

Copyright: American Institute of Physics (AIP) http://www.aip.org/

Postprint available at: Linköping University Electronic Press

http://urn.kb.se/resolve?urn=urn:nbn:se:liu:diva-71638 


\title{
Polaron effects and electric field dependence of the charge carrier mobility in conjugated polymers
}

\author{
Mattias Jakobsson a) and Sven Stafström \\ Department of Physics, Chemistry and Biology (IFM), Linköping University, SE-58183 Linköping, Sweden
}

(Received 23 June 2011; accepted 11 September 2011; published online 6 October 2011)

\begin{abstract}
Charge transport in conjugated polymers has been investigated using Monte Carlo simulations implemented on top of the Marcus theory for donor-acceptor transition rates. In particular, polaron effects and the dependency of the mobility on the temperature and the applied electric field have been studied. The conclusions are that while the qualitative temperature dependence is similar to that predicted by Miller-Abrahams theory in the Gaussian disorder model (GDM), the electric field dependence is characterized by a crossover into the Marcus inverted region, not present in the GDM. Furthermore, available analytical approximations to describe the electric field dependence of the mobility in Marcus theory fail to fit the simulation data and hence cannot be used to directly draw conclusions about the importance of polaron effects for charge transport in conjugated polymers. (c) 2011 American Institute of Physics. [doi:10.1063/1.3644931]
\end{abstract}

\section{INTRODUCTION}

Charge transport in organic materials, such as conjugated polymers, has been extensively studied ever since Heeger, MacDiarmid, and Shirakawa found an astonishingly high conductivity in polyacetylene films oxidized by iodine vapor, ${ }^{1}$ a discovery which eventually lead to the Nobel prize in chemistry. The reason for the interest in conjugated polymers was the often superior processability and mechanical properties of these organic materials compared to metals and the joint research efforts made to study and understand the charge transport process lead to a variety of applications, such as organic light emitting diodes, ${ }^{2,3}$ organic field-effect transistors, ${ }^{4}$ and solar cells. ${ }^{5}$

In any disordered material where the charge transport occurs below the mobility edge, i.e., when the charge carriers move by direct tunneling between localized states instead of occupying extended states as in crystalline materials, the charge transport process is of hopping type. ${ }^{6}$ To theoretically find the transport coefficients of materials characterized by a hopping type of charge transport, the transition rates between the localized states or sites are needed. These are usually taken from Miller-Abrahams ${ }^{7}$ or Marcus ${ }^{8}$ theory, the latter if polarization and/or geometrical reorganization effects (polaron effects) must be accounted for.

If polaron effects can be neglected, the simpler MillerAbrahams transition rate $^{7}$ can be used. Given two sites $i$ and $j$ in a system of absolute temperature $T$, the transition rate for an electron moving from $i$ to $j$ is

$$
k_{i j}=\kappa_{0} \exp \left(-\frac{\varepsilon_{j}-\varepsilon_{i}+\left|\varepsilon_{j}-\varepsilon_{i}\right|}{2 k T}\right),
$$

where $\kappa_{0}$ is the transition rate in the absence of an energy barrier, $k$ is the Boltzmann constant, and $\varepsilon_{i}$ and $\varepsilon_{j}$ is the energy of site $i$ and $j$, respectively. If the distance between site $i$ and $j$

\footnotetext{
a)Electronic mail: matja@ifm.liu.se.
}

is $R_{i j}$, then an eventual contribution from an external electric field parallel to this distance, $E$, enters in Eq. (1) as

$$
\varepsilon_{j}-\varepsilon_{i}=\varepsilon_{j}^{(0)}-\varepsilon_{i}^{(0)}-e E R_{i j}
$$

where $e$ is the elementary charge and the index (0) indicates the site energy in the absence of an external electric field. A critical assumption in Eq. (1) is that for hops downward in energy $\left(\varepsilon_{i}>\varepsilon_{j}\right)$, the exponential factor is equal to unity and the transition rate is simply $\kappa_{0}$ which, in general, is assumed to be independent of both temperature and applied electric field. An implication of this is that the mobility, defined as

$$
\mu=\frac{\langle v\rangle}{E},
$$

becomes independent of the temperature and decreases linearly with $1 / E$ for large fields, since the mean velocity of the charge carriers, $\langle v\rangle$, will have no temperature or field dependence.

To be able to use Eq. (1), the density of states (DOS) of the site energies is needed. For organic disordered materials, the DOS is in general assumed to have a Gaussian distribution. This assumption is known as the Gaussian disorder model (GDM) and was developed by Bässler. ${ }^{9}$ There are some indirect experimental results in support of a Gaussian DOS, but the main reason for the trust the GDM has received is its ability to explain a wide range of experimental observations related to charge transport in molecularly doped polymers and conjugated polymers. ${ }^{9-15}$ A characteristic feature of the GDM is a temperature and applied electric field dependence of the mobility, given by

$$
\mu \propto \exp \left[-\left(C \frac{\sigma}{k T}\right)^{2}\right],
$$

and

$$
\mu \propto \exp \left[\left(\frac{E}{E_{0}}\right)^{1 / 2}\right]
$$


respectively. Here, $\sigma$ is the standard deviation of the Gaussian distribution of site energies (the DOS), $C$ is a constant usually assigned the value $2 / 3$, and $E_{0}$ is a prefactor dependent on $T$. The Poole-Frenkel-like expression given by Eq. (5) is found to be valid for intermediate field strengths but not for very low or high fields. ${ }^{9,13}$ At low fields the transport process can be considered to be a diffusive process and for high fields the discussion concerning the high field mobility (see above) applies. Hence, as the field is increased, the charge transport is said to go from being (1) diffusive to (2) fieldassisted, associated with a Poole-Frenkel field dependence, to (3) field-saturated, where the mobility decrease as $1 / E$.

When polaron aspects are believed to affect the charge transport, Eq. (1) should be replaced by the semi-classical Marcus equation, ${ }^{8}$

$$
k_{i j}=\frac{2 \pi}{\hbar}\left|J_{i j}\right|^{2} \frac{1}{\sqrt{4 \pi \lambda k T}} \exp \left[-\frac{\left(\varepsilon_{j}-\varepsilon_{i}+\lambda\right)^{2}}{4 \lambda k T}\right],
$$

where $\hbar$ is the Planck constant divided by $2 \pi, J_{i j}$ is the transfer integral between site $i$ and $j$, and $\lambda>0$ is the reorganization energy. In Marcus theory, a transition downward in energy will increase the transition rate as long as the difference is not greater than the reorganization energy, i.e., as long as $\varepsilon_{j}-\varepsilon_{i}>-\lambda$. If this condition is not fulfilled, the charge transport is said to be in the Marcus inverted region and the mean velocity of the charge carriers will decrease with increasing field strength. Hence, for large fields, Marcus theory also predicts that the mobility will decrease with increasing field strength, but more rapidly than predicted by Miller-Abrahams theory.

Several attempts have been made to find a closed-form analytical expression for the mobility based on Marcus theory. Verbeek $^{16}$ proposed a 1D first-order approximation given by

$$
\mu=\frac{k_{0} r}{E}\left[1-\exp \left(-\frac{e E r}{k T}\right)\right] \exp \left[-\frac{(-e E r+\lambda)^{2}}{4 \lambda k T}\right],
$$

where $k_{0}$ is the mean transition rate between the sites when $e E r=\lambda$. This expression assumes that the charge transport is in the nearest neighbor regime and that the transport sites are equidistant with a separation $r$, which is an arguable assumption for charge transport in disordered organic materials. Van der Auweraer et al. ${ }^{17}$ improved this expression by applying it to a 3D system. Defining $\Omega=\lambda / k T$ and $W=e E r / k T$, he expressed the mobility as

$$
\begin{aligned}
\mu= & \frac{r \Omega k_{0}}{E W^{2}}\left\{\exp \left[-\frac{(\Omega+W)^{2}}{4 \Omega}\right]-\exp \left[-\frac{(\Omega-W)^{2}}{4 \Omega}\right]\right. \\
& \left.+\frac{1}{2} \sqrt{\Omega \pi}\left[\operatorname{erf}\left(\frac{\Omega+\mathrm{W}}{2 \sqrt{\Omega}}\right)-\operatorname{erf}\left(\frac{\Omega-\mathrm{W}}{2 \sqrt{\Omega}}\right)\right]\right\} .
\end{aligned}
$$

The major difference from Eq. (7) is that Eq. (8) decreases slower with the applied electric field in the Marcus inverted region, although still faster than $1 / E$ as in Miller-Abrahams theory. The reason for this is that in the 3D case when $e E r$ becomes greater than $\lambda$, the transitions have the possibility to take place at an angle to the direction of the field and thereby effectively reducing $r$ to decrease the value of $e E r$. Another analytical expression was derived by Seki and Tachiya ${ }^{18}$ who presented very elaborate calculations that produced an exact analytical solution for the mobility of charge carriers governed by Marcus theory. However, this was still done in the 1D nearest neighbor approximation and should suffer from the same problem Eq. (7) exhibit in the Marcus inverted region.

The attempts ${ }^{13,16,17,19-21}$ that have been made to explain a maximum or decreasing mobility for an increasing field $^{12,13,20,22,23}$ in disordered organic materials as an effect of Marcus theory and its inverted region have in many cases been abandoned in favor of the disorder model. ${ }^{11,12,20,23,24}$ The common reason for this is that the parameters needed to fit the analytical expressions given by Eqs. (7) and (8) fall outside the interval of physically reasonable values and therefore, in many cases, the conclusions have been that polaron effects do not play an important role for charge transport in conjugated polymers.

In this work, we compare the analytical approximations to Monte Carlo simulations of charge transport in conjugated polymers directly based on Marcus theory. The Monte Carlo method, often dubbed as an ideal experiment, allow us to be sure that we observe the effects of Marcus theory, i.e., polaron effects. It has been argued above and by Auweraer et al. ${ }^{17}$ that the Marcus inverted region is not well described in a 1D system or a simple cubic lattice. Hence, in this work we construct a 3D system with aligned, but otherwise random, polymers were the charge carriers are allowed to make transitions within and between the polymer chains. This polymer structure and the Monte Carlo method we use are explained in Sec. II. In Sec. III, the results of the simulations, including attempts to fit it to closed-form analytical expressions, are presented together with an extensive discussion. This is followed by a short summary in Sec. IV.

\section{METHOD}

In order to study hopping charge transport in disordered conjugated polymers, we have implemented a simulation scheme based on the dynamic variable step size Monte Carlo method developed by Bässler. ${ }^{9}$ This implementation includes constructing a geometrical structure of sites (chromophores), creating couplings between them, and finally running the simulation by observing a random walk of a charge carrier moving between these sites over the couplings. In this work, we consider electrons as charge carriers and limit the studies to the low concentration regime, i.e., there is no interaction between charge carriers in the simulations.

First we introduce a strongly anisotropic spatial structure representing aligned polymers, replacing the simple cubic lattice of sites used by Bässler. When material parameters are needed to model the polymers, they are explicitly referenced in what follows and taken from studies of poly(p-phenylene vinylene) (PPV). The polymer chains are built up from a number of chromophores and each such chromophore consists of a certain number of monomers. The electronic wave functions are assumed to be localized to the individual chromophores, resulting in the need for a charge carrier to tunnel between them in order to propagate through the system. Each chromophore has an associated intrinsic energy and size, 
(a)

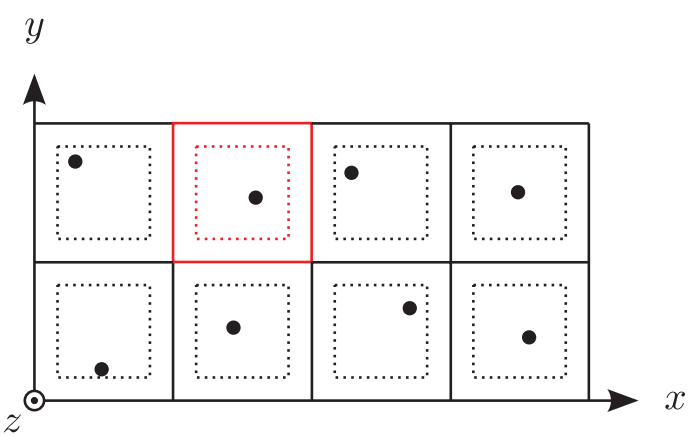

(b)

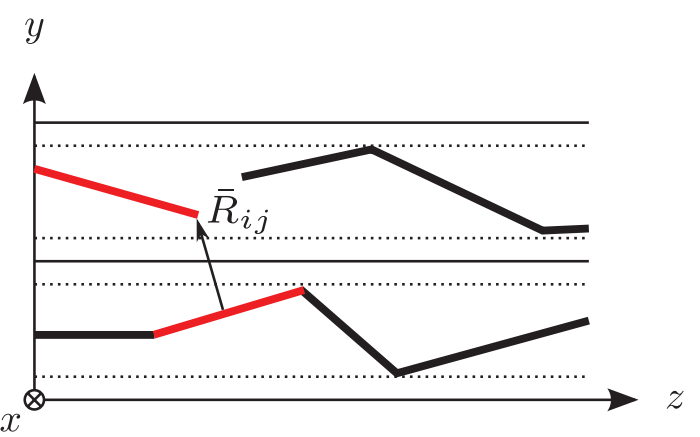

FIG. 1. A cross section of the polymer structure in (a) the $x y$ plane and (b) the $y z$ plane, illustrating the cell grid used to generate it. In part (a), a cell and its inner bounding box are marked in red. In part (b), two chromophores in different polymers are marked in red and the shortest possible vector connecting them is drawn in the figure. The length of this vector, $R_{i j}$, is used in Eq. (9).

where the size is the number of monomers it contains. Both these attributes are used to calculate the energy of the chromophore's orbitals.

To generate the polymer structure, a cell grid is created in the $x y$ plane according to Fig. 1(a). For any given $z$ coordinate, each cell can contain at most one polymer and this polymer must be located within an inner bounding box shown as a dotted line in each cell in the figure. This bounding box is enforced to guarantee a finite minimum distance between the polymers. Given these constraints, polymers extending in the $z$ direction consisting of a sequence of chromophores are generated at random within each cell, according to the following procedure.

Starting at $z=0$, an $x$ and $y$ coordinate is drawn from a uniform distribution within the inner bounding box of the current cell. These three coordinates mark the start of the polymer and hence the start of the first chromophore within the polymer. Next, the number of monomers in the first chromophore is drawn from a random integer distribution and a new $x$ and $y$ coordinate is drawn within the inner bounding box to mark the other end of the chromophore. The $z$ coordinate of this end is chosen so that the length of the chromophore corresponds to the number of already drawn monomers. This end is now used as the start of the next chromophore and the procedure is repeated until the polymer contains a predetermined number (see below) of chromophores.

The polymers generated within a cell are separated by a finite distance in the $z$ direction, drawn from yet another ran- dom distribution. Finally, when all polymers in the cell have been generated, the whole cell is translated a random distance in the $z$ direction so that all cells do not contain a polymer starting at $z=0$. The parameters used to generate the polymer structures in the simulations are 15 polymers per cell, 40 chromophores per polymer, and 5-10 monomers per chromophore drawn from a discrete uniform distribution. A sample of the end result is shown in Fig. 1(b).

When all the chromophores have been generated, they are coupled to each other. This is done by going through each chromophore, finding all other chromophores within a certain cutoff distance and assigning a transition rate between them. The cutoff distance is carefully chosen so that it is large enough not to affect the outcome of the simulation. In the simulations presented in this article, a cutoff distance of $20 \AA$ is used. Note that this is a cutoff of the distance between the two closest points of the chromophores and that the center-tocenter distance for the chromophores may by far exceed this value. During the coupling phase, periodic boundary conditions are imposed. In the $x$ and $y$ direction, the periodic distance is simply the number of columns and rows of cells, respectively, multiplied by the side length of each cell. In the $z$ direction, each cell has its own spatial period depending on the total length of the polymers generated to produce it.

The transition rates between the chromophore states are taken from the semi-classical Marcus theory (Eq. (6)). The electronic transfer integral, $J_{i j}$, depends on the distance, $R_{i j}$, between the donor site $i$ and the acceptor site $j$. For the extended chromophore sites used in the simulations, this distance is defined as the length of the shortest possible vector connecting the chromophores (see Fig. 1(b)). The dependence is assumed to be $\mathrm{be}^{25}$

$$
J_{i j}=\frac{J_{0}}{\sqrt{n_{i} n_{j}}} \exp \left[-\frac{\beta}{2}\left(R_{i j}-R_{0}\right)\right] .
$$

Here, $J_{0}$ is a constant that coincide with the electronic transfer integral between two monomers not subject to form a delocalized state and separated by a distance $R_{0}$. Both these constants are chosen depending on if the transition is between a donor and an acceptor within the same polymer (intra-polymer transition) or not (inter-polymer transition). $n_{i}$ and $n_{j}$ is the number of monomers in the donor and acceptor chromophore, respectively, and the fall-off parameter $\beta$ is related to the electronic localization length, $\alpha$, according to $\beta=2 / \alpha$. This exponential prefactor determines the strength of the distance dependence of Eq. (9). For the special case of nearest neighbor transitions within the same polymer, the transfer integral is simply given by $J_{0} / \sqrt{n_{i} n_{j}}$.

Figure 2 shows the density of the electronic transfer integrals for the possible transitions in the system. The transitions are split into intra-polymer transitions and transitions that occur between different polymers in the same cell (intracell) or different cells (inter-cell). The system is in this case and in the simulations presented in this work defined by chromophores containing 5-10 monomers, electronic transfer integrals of $0.2(0.1) \mathrm{eV}$ at a distance of 0 (2) $\AA$ for intra-polymer (inter-polymer) transitions. The values for the electronic transfer integrals were picked within the interval reported for randomly oriented PPV chains ${ }^{26}$ and kept small 


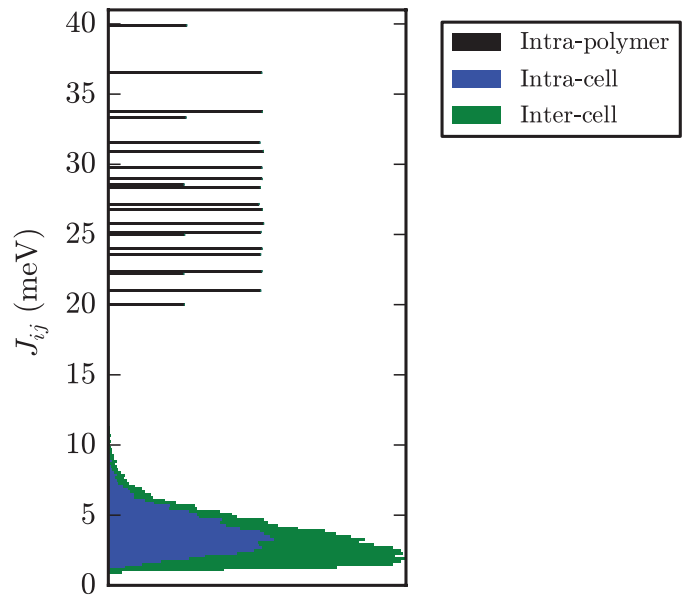

FIG. 2. Off-diagonal disorder in the systems for three different transition types.

enough to ensure localization to each chromophore. The falloff parameter $\beta$ is equal to $0.6 \AA^{-1}$, a value which corresponds to a localization length within the interval reported for $\mathrm{PPV}^{27}$ The figure gives a clear view of the off-diagonal disorder in the system, i.e., the disorder of the electronic transfer integrals with respect to different transitions. The disorder associated with intra-polymer transitions stem from the variation of localization lengths for chromophores of different sizes (different number of monomers), which results in a fairly discrete spectrum. For the weaker inter-polymer couplings, the variation in the distance between the donor and acceptor chromophores dominates the disorder and the result is a skewed Gaussian-shaped continuous spectrum. It can also be deduced from Fig. 2 that intra-polymer transitions, i.e., nearest neighbor chromophore transitions, will be strongly favored if the energy difference between the chromophores (the diagonal disorder) is neglected.

The energy difference in Eq. (6) is just as in MillerAbrahams theory calculated according to Eq. (2). The energies in the absence of an external electric field are calculated according to Hückel theory. Since each chromophore $i$ consists of a number of monomers, $n_{i}$, and the orbitals of these adjacent monomers are assumed to be of roughly equal energy, a splitting into $n_{i}$ states extending over the whole chromophore will occur. The energy of a state $k$ of a chromophore in the absence of an external electric field is then given by

$$
\varepsilon_{i}^{(0)}=\varepsilon_{i, k}=\varepsilon_{0}+2 J_{0} \cos \frac{k \pi}{n_{i}+1},
$$

where $\varepsilon_{0}$ is the energy associated with an isolated monomer of the chromophore and $J_{0}$ is the electronic transfer integral between two adjacent monomers. In the simulations, $\varepsilon_{0}$ is either drawn from a Gaussian distribution to introduce a greater energetic disorder in the system or simply set to zero. For the acceptor, any orbital $k \in\left[1, n_{i}\right]$ is a valid state, while for the donor it is assumed that the charge carrier has enough time until the next transition to relax into the LUMO state of $k=n_{i}$. The final transition rate between a donor and an acceptor chromophore is taken as the sum of the transition rates to all states of the acceptor. The LUMO state of the acceptor will have the highest transition rate and hence be the most

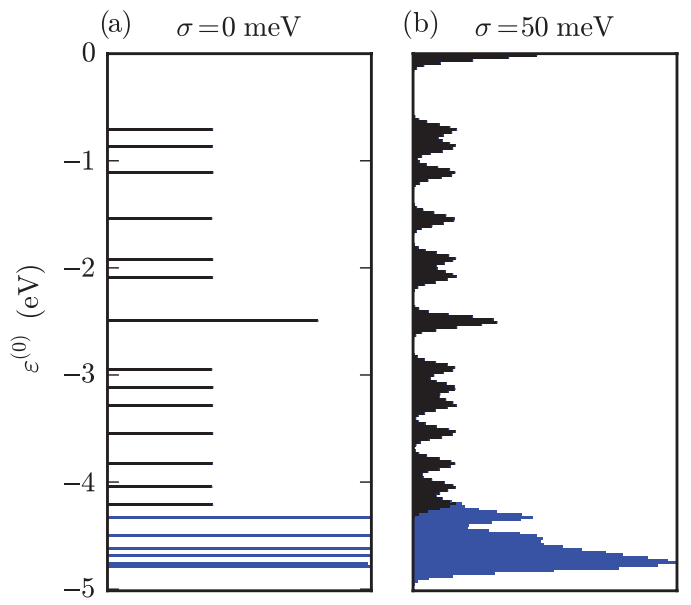

FIG. 3. Diagonal disorder in the systems with a distribution of chromophores containing 5-10 monomers for (a) no extrinsic disorder and (b) a Gaussian disorder of $50 \mathrm{meV}$. The blue bars indicate the LUMO states of the sites.

probable receiving state, but we have previously shown ${ }^{25}$ that the remaining states can play a significant role for the charge transport process and should not be neglected.

Figure 3 shows the density of states for the electrons (or the diagonal disorder) for (a) no applied Gaussian disorder and (b) an applied Gaussian disorder of $50 \mathrm{meV}$. This width of the Gaussian is chosen so that the width of the DOS, after the broadening caused by Eq. (10) applied with different chromophores sizes, $n_{i} \in[5,10]$, is roughly equivalent to a pure Gaussian distribution of width $90 \mathrm{meV}$, as reported for PPV. ${ }^{12}$ The blue bars correspond to the lowest-lying orbitals of the chromophores, i.e., an orbital with an energy in this range is always available, no matter which chromophore is considered. It is worth noting that the Hückel model (Eq. (10)) give rise to a symmetric DOS around zero, but only negative energies are shown in Fig. 3 for clarity.

After the polymer structure is generated and the couplings between the chromophores are established, the Monte Carlo simulation is carried out by inserting a charge carrier at a random site and allowing it to move through the system. Due to the diagonal disorder present in the system, the Monte Carlo simulation of the charge transport can be characterized as variable range hopping. The transition rates are used to find the probabilities for the different transitions at each site and to draw the dwell time, i.e., the time the charge will spend on its current donor until the drawn transition is carried out. After a predetermined time, $\tau_{\text {tot }}$, the distance the charge has traveled, $d$, is measured and used together with the electric field strength, $E$, to find the drift mobility according to

$$
\mu=\frac{d}{\tau_{\mathrm{tot}} E} .
$$

We finally note that when the simulations were carried out, careful consideration was taken to avoid finite size effects of the spatial structure. The number of cells in the grid and the number of chromophores/polymers in one cell is finite, but an infinite sample is created by imposing periodic boundary conditions in all directions. It is important to make the finite structure large enough so that replacing it with another will not have a significant impact on the results of the 
simulation. Extra care was also taken to run the simulations on a large enough ensemble to be able to produce good statistical averages.

\section{RESULTS AND DISCUSSION}

The results presented in this article are from simulations run on in total eight different systems. These systems are given by all possible combinations of (i) a reorganization energy of 0.1 or $0.3 \mathrm{eV}$, (ii) no extrinsic diagonal disorder or an extrinsic Gaussian diagonal disorder of $50 \mathrm{meV}$ (applied to $\varepsilon_{0}$ in Eq. (10)), and (iii) a field applied either in the $z$-direction, i.e., parallel to the general direction of the polymers, or in the $y$-direction, i.e., orthogonal to the polymers. The reorganization energies are taken as a low and high value for those reported for PPV. ${ }^{28}$ Systems with no extrinsic diagonal disorder are used as model systems to better understand the charge transport process, while the systems with extrinsic diagonal disorder are assumed to be closer to a real system. The different electric field directions are treated to study the anisotropy of our polymer structure.

\section{A. Energetic relaxation}

The physical property of main interest in this work is the mobility of the charge carriers. Since we are studying a system characterized by a DOS of discrete energy levels (no extrinsic diagonal disorder) or with a Gaussian disorder applied to these discrete levels, the mean energy of the charge carriers is expected to reach an equilibrium value in the longtime limit. That this is in fact true is made evident in Fig. 4, where the mean energy of the charge carriers is plotted for different temperatures as a function of time for a reorganization energy of $\lambda=0.3 \mathrm{eV}$ and an extrinsic Gaussian diagonal disorder of $\sigma=50 \mathrm{meV}$. The applied electric field is $1 \times 10^{5} \mathrm{~V} / \mathrm{cm}$ and the temperature is varied between $150-400$ $\mathrm{K}$. The DOS from Fig. 3 is included to the right in the figure as a reference. Since the charge carriers are inserted at random chromophores in the system, their initial mean energy will be the mean energy of the LUMO states at around $-4.62 \mathrm{eV}$. It is apparent from the figure that during a certain time interval, known as the relaxation time, the mean energy of the charge carriers drops as they find chromophores with lower and lower energy. After the relaxation time has expired, however, the en-

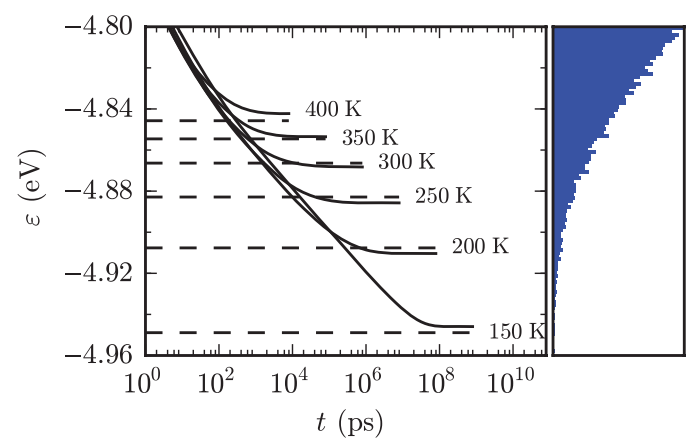

FIG. 4. Energetic relaxation of the charge carriers. The DOS of the system is displayed as a reference to the right.
TABLE I. Parameters to fit the Eq. (12) to the simulated temperature dependence of the longtime limit energy. $\|$ and $\perp$ indicate an electric field of $1 \times 10^{5} \mathrm{~V} / \mathrm{cm}$ applied parallel and orthogonal to the general direction of the polymers, respectively. All systems have an extrinsic Gaussian diagonal disorder of $50 \mathrm{meV}$.

\begin{tabular}{lccc}
\hline \hline \multirow{2}{*}{ System } & & \multicolumn{2}{c}{ Parameters } \\
\cline { 4 - 5 }$\lambda$ & $\vec{E}$ & $\epsilon_{0}$ & $\sigma_{\text {eff }}$ \\
$(\mathrm{eV})$ & $(-)$ & $(\mathrm{eV})$ & $(\mathrm{meV})$ \\
\hline 0.1 & $\|$ & -4.77 & 48.7 \\
0.1 & $\perp$ & -4.78 & 52.6 \\
0.3 & $\|$ & -4.79 & 44.9 \\
0.3 & $\perp$ & -4.78 & 52.3 \\
\hline \hline
\end{tabular}

ergy becomes time-independent and the charge transport has a well-defined equilibration energy, $\varepsilon_{\infty}$. Figure 4 also shows that this relaxation time increases exponentially as the temperature of the system decreases, as is expected. ${ }^{9}$

Once the charge carriers mean energy reaches the equilibration value, the charge transport is in a dynamic equilibrium and the kinetic coefficients including the mobility can be unambiguously determined. Hence, every simulation run to find the mobility of the charge carriers is first required to complete this relaxation and it can be noted that the exponential dependence of the relaxation time on the temperature is the main computational limitation of the simulations.

The mean energy in the longtime limit for the systems with extrinsic diagonal disorder can be fitted to the expression

$$
\varepsilon_{\infty}=\lim _{t \rightarrow \infty} \varepsilon=\epsilon_{0}-\frac{\sigma_{\mathrm{eff}}^{2}}{k_{B} T},
$$

where the values of the parameters $\epsilon_{0}$ and $\sigma_{\text {eff }}$ are listed in Table I. This is clearly shown in Fig. 5, where the longtime limit mean energy of the charge carriers is plotted as a function of the absolute temperature for all systems with an extrinsic Gaussian diagonal disorder. The circles/triangles correspond to a reorganization energy of $0.1 / 0.3 \mathrm{eV}$ and a black/blue marker corresponds to an applied electric field parallel/orthogonal to the general direction of the polymers. The strength of the field is $1 \times 10^{5} \mathrm{~V} / \mathrm{cm}$ for all simulations and the solid lines correspond to Eq. (12) used with the parameters in Table I.

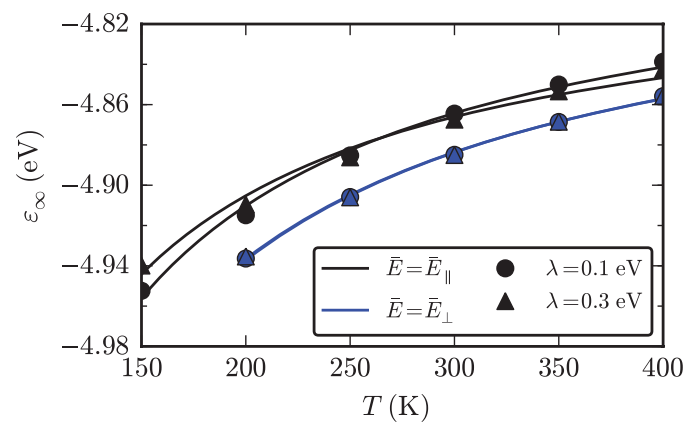

FIG. 5. The longtime limit energy of the charge carriers as a function of temperature for systems with an extrinsic Gaussian diagonal disorder of $50 \mathrm{meV}$ and an applied electric field strength of $1 \times 10^{5} \mathrm{~V} / \mathrm{cm}$. The lines show Eq. (12) fitted to the data points. 


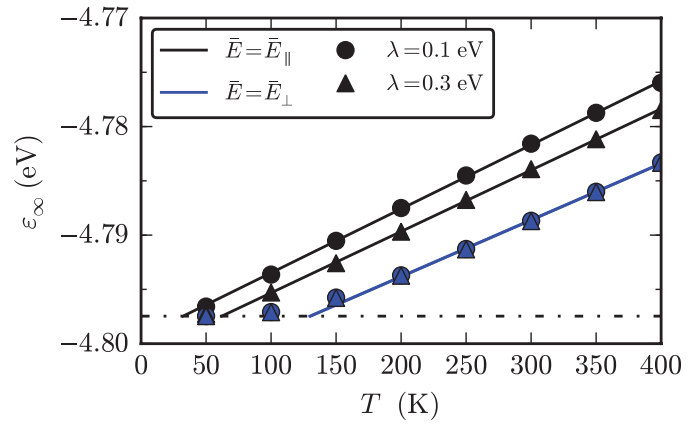

FIG. 6. The longtime limit energy of the charge carriers as a function of temperature for systems with no extrinsic diagonal disorder and an applied electric field strength of $1 \times 10^{5} \mathrm{~V} / \mathrm{cm}$. The energy of the LUMO states is marked by the dashed-dotted line and the solid lines are linear fits to the data points.

For a pure Gaussian DOS, Eq. (12) can be derived analytically ${ }^{9}$ and $\epsilon_{0}$ and $\sigma_{\text {eff }}$ is the mean and the standard deviation of the Gaussian, respectively. In our case, $\epsilon_{0}$ is very close to the unperturbed energy level of chromophores of ten monomers, the largest available chromophores and hence the lowest in energy, and $\sigma_{\text {eff }}$ is very close to the standard deviation of the applied Gaussian disorder to this level. This is in accordance with the reasonable assumption that as the charge carriers relax, they will spend most of their time in the tail states of the largest chromophores due to the favorable energy of their orbitals.

In Fig. 6, the longtime limit energies of the charge carriers are plotted against the absolute temperature for the model systems with no extrinsic diagonal disorder. Here, the temperature dependence is no longer described by Eq. (12), but a clear linear dependence is seen above some threshold energy closely above the LUMO energy of the ten monomer chromophores marked by the dashed-dotted line. The slope is given by about $0.058 \mathrm{meV} / \mathrm{K}$ for systems with an electric field applied parallel to the polymers and $0.052 \mathrm{meV} / \mathrm{K}$ for an orthogonal field. Figure 6 also shows that as the temperature drops, the mean charge carrier energy approaches the ten monomer LUMO energy, i.e., the charge carriers will almost exclusively occupy the ten monomer chromophores and the charge transport process will be carried out in the extreme variable range hopping regime. This is true regardless of the other parameters of the system.

\section{B. Temperature dependence of the charge carrier mobility}

In Fig. 7, the mobility is plotted as a function of the inverse absolute temperature for the model systems in Fig. 6 and an applied electric field of $1 \times 10^{5} \mathrm{~V} / \mathrm{cm}$. The temperature dependence is well described by the Arrhenius equation,

$$
\mu(T)=\mu_{0} \exp \left(-\frac{\Delta}{k_{B} T}\right)
$$

where the least squares fitted values of the parameters $\mu_{0}$ and the activation energy $\Delta$ is available in Table II. The anisotropy of the polymer structure is made apparent by noting that

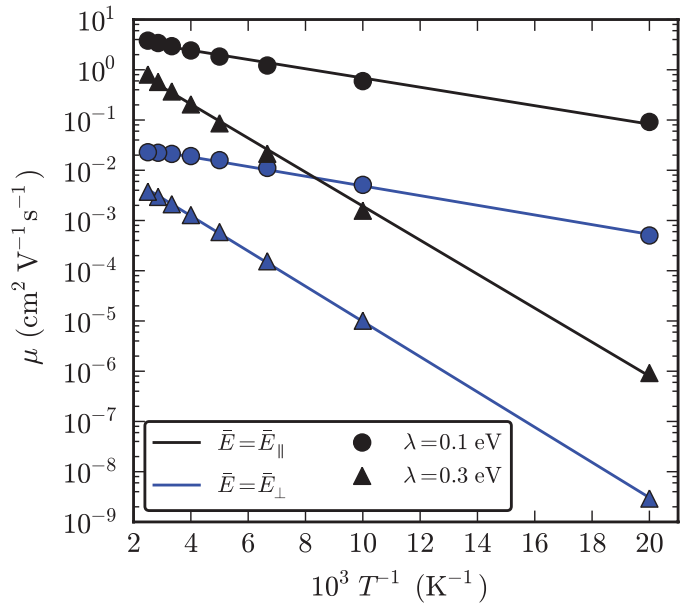

FIG. 7. The mobility as a function of the inverse absolute temperature for systems with no extrinsic diagonal disorder and an applied electric field strength of $1 \times 10^{5} \mathrm{~V} / \mathrm{cm}$. The lines show the Arrhenius equation (Eq. (13)) fitted to the data points.

changing the direction of the applied electric field from parallel to the polymers to orthogonal induces a large drop in mobility, fairly constant with respect to the temperature. Figure 7 and Table II also show that an increase of the reorganization energy results in an increase in the activation energy, which is natural as long as we are not in the Marcus inverted region. This occurrence will be discussed in more detail below.

Figure 8 shows the mobility as a function of the inverse absolute temperature for the systems in Fig. 5 with an extrinsic Gaussian diagonal disorder of $50 \mathrm{meV}$. Two values of the electric field strength are available for each different system and the value is noted next to the lines in the plot. The lines show either the Arrhenius equation (Eq. (13)) or Bässler's equation (Eq. (4)) depending on which expression has the better least squares fit.

For an electric field applied parallel to the polymers and with a strength of $1 \times 10^{5} \mathrm{~V} / \mathrm{cm}$, the temperature dependence is still best described by the Arrhenius equation (Eq. (13)) and the fitted parameters can be found in Table II. The trend from the model systems still holds, increasing the reorganization energy increases the activation energy. Furthermore, we note that applying a Gaussian diagonal disorder also increases the

TABLE II. Parameters to fit the Arrhenius equation (Eq. (13)) to the simulated temperature dependence of the mobility. $\|$ and $\perp$ indicate an electric field of $1 \times 10^{5} \mathrm{~V} / \mathrm{cm}$ applied parallel and orthogonal to the general direction of the polymers, respectively.

\begin{tabular}{|c|c|c|c|c|}
\hline \multicolumn{3}{|c|}{ System } & \multicolumn{2}{|c|}{ Parameters } \\
\hline $\begin{array}{l}\lambda \\
(\mathrm{eV})\end{array}$ & $\begin{array}{c}\sigma \\
(\mathrm{meV})\end{array}$ & $\begin{array}{c}\vec{E} \\
(-)\end{array}$ & $\begin{array}{c}\mu_{0} \\
\left(\mathrm{~cm}^{2} \mathrm{~V}^{-1} \mathrm{~s}^{-1}\right)\end{array}$ & $\begin{array}{c}\Delta \\
(\mathrm{meV})\end{array}$ \\
\hline 0.1 & 0 & $\|$ & 5.69 & 18.2 \\
\hline 0.1 & 0 & $\perp$ & 0.0448 & 19.1 \\
\hline 0.3 & 0 & $\|$ & 4.68 & 67.2 \\
\hline 0.3 & 0 & $\perp$ & 0.0312 & 69.5 \\
\hline 0.1 & 50 & $\|$ & 23.2 & 86.2 \\
\hline 0.3 & 50 & $\|$ & 21.2 & 136 \\
\hline
\end{tabular}




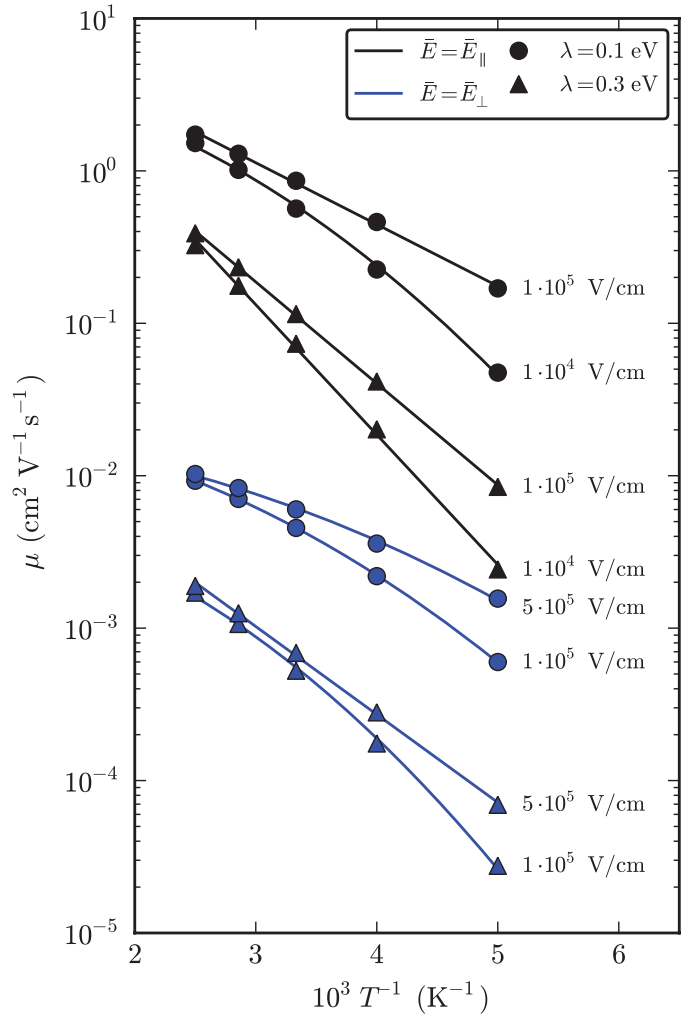

FIG. 8. The mobility as a function of the inverse absolute temperature for systems with an extrinsic Gaussian diagonal disorder of $50 \mathrm{meV}$. The strength of the applied electric field is noted next to the data sets. The lines show the better fit of either the Arrhenius equation (Eq. (13)) or Bässler's equation (Eq. (4)) to the data points.

activation energy, as is expected. For an electric field with the same strength but applied orthogonal to the polymers, however, the temperature dependence is better described by the relationship found for the GDM given by Eq. (4). The parameters to fit this equation to the simulation data points can be found in Table III, where $\mu_{0}$ is the proportionality constant and $C$ is set to $2 / 3$.

The agreement with the GDM temperature dependence found for orthogonal fields is not surprising considering the fact that we have applied a Gaussian diagonal disorder to our systems. It is instead more surprising that the simulations for a parallel electric field fit the Arrhenius behavior better, even though there is an applied Gaussian disorder in these systems as well. One explanation is that the electric field strength is too high to show the GDM behavior in the parallel case, which is

TABLE III. Parameters to fit the Bässler equation (Eq. (4)) to the simulated temperature dependence of the mobility. The constant $C$ is set to $2 / 3 . \perp$ indicates an electric field of $1 \times 10^{5} \mathrm{~V} / \mathrm{cm}$ applied orthogonal to the general direction of the polymers.

\begin{tabular}{|c|c|c|c|c|}
\hline \multicolumn{3}{|c|}{ System } & \multicolumn{2}{|c|}{ Parameters } \\
\hline $\begin{array}{l}\lambda \\
(\mathrm{eV})\end{array}$ & $\begin{array}{c}\sigma \\
(\mathrm{meV})\end{array}$ & $\begin{array}{c}\vec{E} \\
(-)\end{array}$ & $\begin{array}{c}\mu_{0} \\
\left(\mathrm{~cm}^{2} \mathrm{~V}^{-1} \mathrm{~s}^{-1}\right)\end{array}$ & $\begin{array}{c}\sigma \\
(\mathrm{meV})\end{array}$ \\
\hline 0.1 & 50 & $\perp$ & 0.0231 & 49.4 \\
\hline 0.3 & 50 & $\perp$ & 0.00632 & 60.5 \\
\hline
\end{tabular}

evident for a reorganization energy of $0.1 \mathrm{eV}$. For this system, lowering the applied electric field to $1 \times 10^{4} \mathrm{~V} / \mathrm{cm}$ shifts the temperature dependence from the Arrhenius type to the GDM. This is, however, not the case for a reorganization energy of $0.3 \mathrm{eV}$, where the Arrhenius behavior remains even at the low field of $1 \times 10^{4} \mathrm{~V} / \mathrm{cm}$. Similarly, for an orthogonal electric field, increasing the strength to $5 \times 10^{5} \mathrm{~V} / \mathrm{cm}$ shifts the system with a reorganization energy of $0.3 \mathrm{eV}$ from the GDM to the Arrhenius dependence, but for the system with a reorganization energy of $0.1 \mathrm{eV}$ this shift does not occur. It is evident that while the strength of the electric field is important to show the GDM temperature dependence, other properties of the system play a crucial role as well.

A trend from Fig. 8 is that the systems where the electric field is applied parallel to the polymers are more prone to show the Arrhenius temperature dependence. One possible explanation for this is that a parallel field strongly favors intra-polymer transitions and, in particular, nearest neighbor hopping due to both spatial proximity and a field-induced energetic advantage. In the extreme case of only nearest neighbor transitions, the charge carriers utilize the whole DOS seen in Fig 3(b) since it cannot avoid the high energy states. This superposition of Gaussian distributions is far from a pure Gaussian DOS and there is no reason to expect a temperature dependence described by Eq. (4). On the other hand, an orthogonal field will make inter-cell transitions (inter-polymer transitions between different cells) more favorable and there are, in general, a larger number of plausible transitions in this direction, where the one with the most favorable energy can be chosen. This means that the charge carriers will be more subject to variable range hopping and avoid the high energy states of Fig. 3(b). They will instead mostly reside in the tail states of the DOS that closely resemble the tail states of a pure Gaussian and hence the temperature dependence should be similar to that of the GDM. In support of this theory, Fig. 5 shows that rotating the field from parallel to orthogonal results in a significant drop in the mean energy of the charge carriers, which according to the discussion above should reflect a transition from nearest neighbor hopping to a more variable range hopping-like transport.

A final note should be made regarding the high mobilities in Fig. 8 and all plots of the mobility in what follows for an electric field applied parallel to the polymers at room temperature. This is a result of the long extended localized states along the polymers, all mutually aligned head to tail and in the same direction. In a real system, the polymers will not exhibit this type of alignment and the mobilities are considerably lower.

\section{Electric field dependence of the charge carrier mobility}

Figure 9 shows the mobility as a function of the strength of an electric field applied parallel to the general direction of the polymers at a temperature of $300 \mathrm{~K}$. Panels (a) and (b) show simulations run with a reorganization energy of $0.1 \mathrm{eV}$ and panels (c) and (d) correspond to a reorganization energy of $0.3 \mathrm{eV}$. Panels (a) and (c) show the model system with no extrinsic diagonal disorder and panels (b) and (d) 


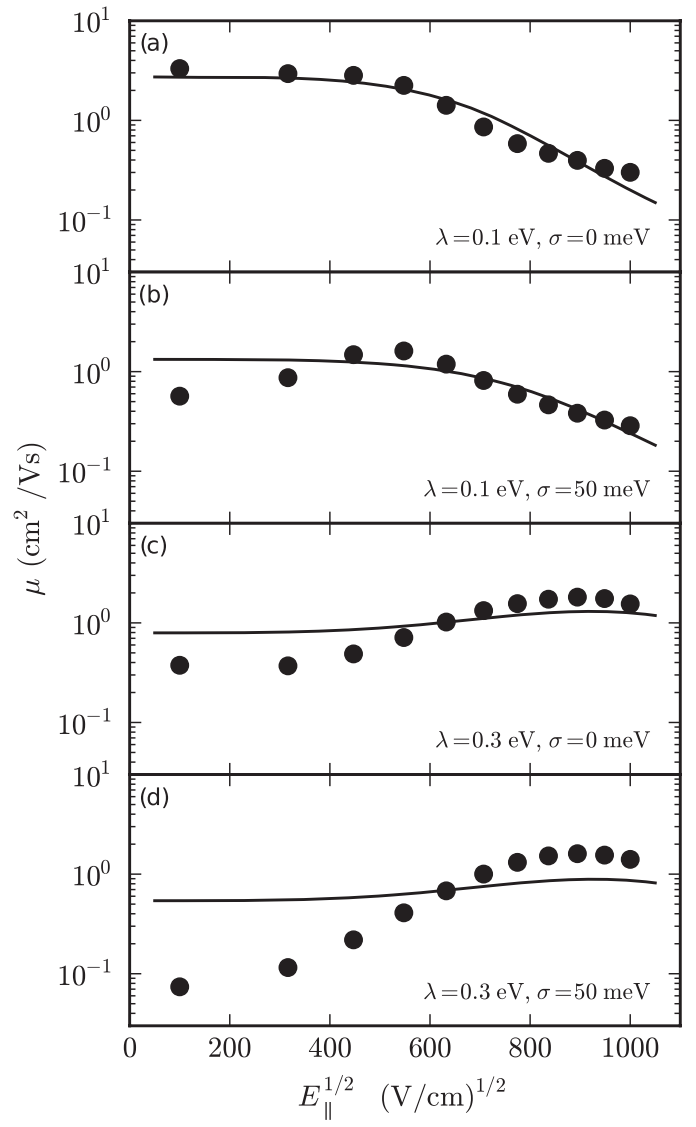

FIG. 9. The mobility as a function of an electric field applied parallel to the general direction of the polymers at a temperature of $300 \mathrm{~K}$. The lines show the Auweraer expression (Eq. (8)) fitted to the data points.

show the system with an extrinsic Gaussian diagonal disorder of $50 \mathrm{meV}$.

The qualitative behavior of the electric field dependence, characterized by an increasing mobility with the electric field followed by a maximum and then a decreasing mobility, is in accordance with that found in previously published experimental results. ${ }^{13,16,20,23}$ In our case, it is certain that the observed maximum arise from a crossover into the Marcus inverted region, since the theory is defined explicitly in the implementation of the Monte Carlo simulation. It is also made apparent from the shift of the maximum to a higher applied electric field when the reorganization energy is increased (compare Figs. 9(a) and 9(b) with Figs. 9(c) and 9(d)). The lines in Fig. 9 show the Van der Auweraer expression (Eq. (8)) fitted to the data points using a modified LevenbergMarquardt least squares method. The parameters used are listed in Table IV. Even though Eqs. (7) and (8) can qualitatively follow the simulated data points, they cannot be fitted to them in a satisfactory way, since in all the cases the simulation results exhibit a stronger electric field dependence than the approximate equations. This is also in accordance with prior observations. ${ }^{20}$

Figure 10 shows the electric field dependence of the mobility when the field is applied orthogonal to the polymers at $300 \mathrm{~K}$. From top to bottom, the order of the simulation parameters is the same as in Fig. 9, but note that Fig. 10 has a continuous y-axis. In general, the electric field dependence is
TABLE IV. Parameters to least squares fit the Auweraer expression (Eq. (8)) to the simulated electric field dependence of the mobility at a temperature of $300 \mathrm{~K}$. $\|$ and $\perp$ indicate an electric field applied parallel and orthogonal to the general direction of the polymers, respectively.

\begin{tabular}{lcccc}
\hline \hline & System & & \multicolumn{2}{c}{ Parameters } \\
\cline { 5 - 6 }$\lambda$ & $\sigma$ & $\vec{E}$ & $k_{0}$ & $r_{0}$ \\
$(\mathrm{eV})$ & $(\mathrm{meV})$ & $(-)$ & $\left(\mathrm{ps}^{-1}\right)$ & $(\AA)$ \\
\hline 0.1 & 0 & $\|$ & 8.19 & 36.9 \\
0.1 & 50 & $\|$ & 7.41 & 27.0 \\
0.3 & 0 & $\|$ & 20.0 & 33.5 \\
0.3 & 50 & $\|$ & 14.0 & 33.1 \\
0.1 & 0 & $\perp$ & 59.1 & 1.22 \\
0.1 & 50 & $\perp$ & 30.8 & 0.914 \\
0.3 & 0 & $\perp$ & 0.278 & 14.6 \\
0.3 & 50 & $\perp$ & 0.0219 & 26.8 \\
\hline \hline
\end{tabular}

weaker in the orthogonal case compared to the parallel, especially for the systems with no extrinsic diagonal disorder. Furthermore, only the system with a reorganization energy of $0.1 \mathrm{eV}$ and no extrinsic diagonal disorder exhibit a maximum (barely visible on the scale in Fig. 10) in the mobility in the range of field strengths under study. This is because of a much shorter transition distance (see discussion above) in the direction of the electric field that reduces the field-dependent term $e E r$ in the activation energy compared to the reorganization energy. Hence, the field never gets large enough for $e E r$ to become greater than the $\lambda$ and to enter into the Marcus inverted region. This is also an explanation for the weaker field dependence; a generally smaller $r$ results in a smaller variation in the activation energy under the interval of field strengths studied and hence a smaller variation in mobility.

As in Fig. 9, the lines in Fig. 10 correspond to the Auweraer equation. The parameters obtained from least squares fitting are available in Table IV and studying the $r$ column confirms the generally shorter transition distance in the orthogonal case. For a reorganization energy of $0.1 \mathrm{eV}$, Eq. (8) has no maximum and is a strictly decreasing function with respect to the field, regardless of the $r$ parameter. This is contrary to the simulation results, that exhibit a maximum in the case of no extrinsic diagonal disorder or is strictly increasing with respect to the applied electric field for a Gaussian diagonal disorder of $50 \mathrm{meV}$. A better fit is obtained for a reorganization energy of $0.3 \mathrm{eV}$ and, in particular, for the case of no extrinsic diagonal disorder. For the system with a Gaussian diagonal disorder of $50 \mathrm{meV}$, however, the field dependence is once again stronger in the simulations than the analytical approximation can follow.

\section{SUMMARY AND CONCLUSIONS}

In this work, we have studied charge transport in conjugated polymers using Monte Carlo simulations. In particular, we have investigated polaronic effects by describing the charge transport using Marcus theory. We show that with regard to temperature, the qualitative behavior of the charge transport process is not notably affected compared to a nonpolaron description using Miller-Abrahams theory. This is 


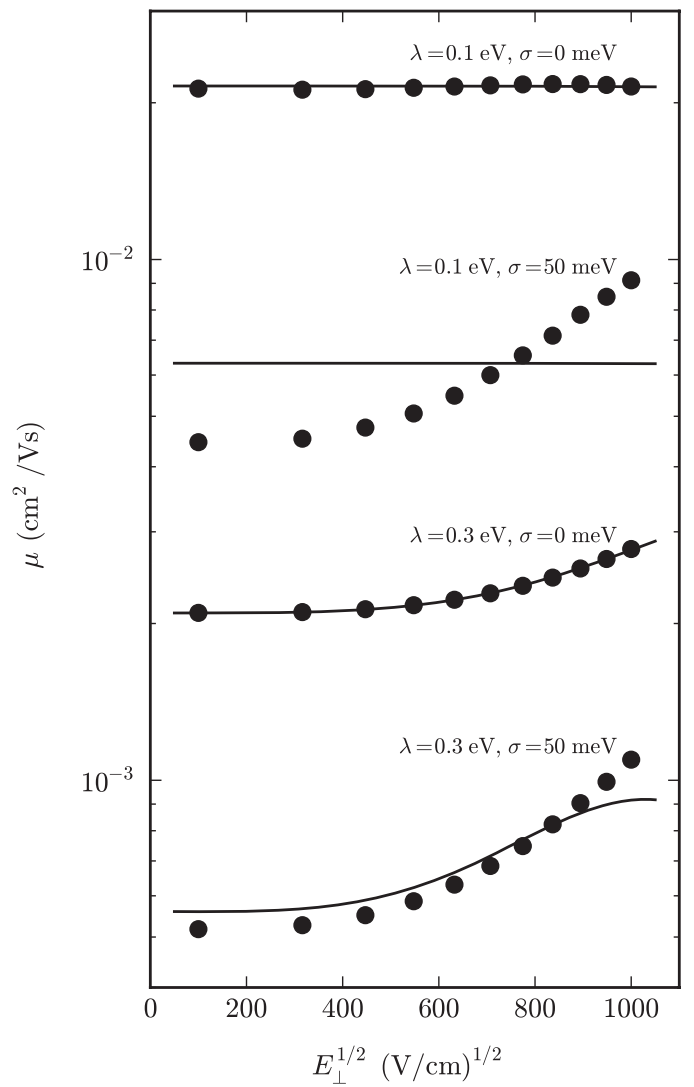

FIG. 10. The mobility as a function of an electric field applied orthogonal to the general direction of the polymers at a temperature of $300 \mathrm{~K}$. The lines show the Auweraer expression (Eq. (8)) fitted to the data points.

based on the observations that (1) the long-time limit mean energy of the charge carriers follow a $1 / T$ dependence (see Eq. (12)) and (2) the temperature dependence of the mobility varies exponentially with $-1 / T^{2}$ (see Eq. (4)). Both these dependencies are also observed for the GDM and MillerAbrahams theory. Thus, the temperature dependence is not conclusive whether or not polarons play a role for charge transport in conjugated polymers.

The simulated electric field dependence of the mobility exhibits a maximum, a dependence that has been observed in the experimental work as well. ${ }^{13,16,20,23}$ The origin of this maximum has been debated and a crossover into the Marcus inverted region is one possible explanation. However, the failure to fit analytical approximations derived from Marcus theory to the experimental results has lead researchers to discard the polaron theory in favor of the GDM. In this work, we show that the analytical expressions fail to describe the electric field dependence of the mobility produced by Monte Carlo simulations directly based on Marcus theory. We are also able to show that the Marcus theory results in a field dependence of the mobility which is in qualitatively good agreement with the experimental data. ${ }^{13,16,20,23}$ In order to be conclusive with respect to the importance of polarons, the next step would be to derive better analytical expressions.

\section{ACKNOWLEDGMENTS}

Financial support from the Swedish Research Council (VR) and computational resources and support from the MATTER Network is gratefully acknowledged.

${ }^{1}$ H. Shirakawa, E. J. Louis, A. G. MacDiarmid, C. K. Chiang, and A. J. Heeger, J. Chem. Soc., Chem. Commun. 578 (1977).

${ }^{2}$ C. W. Tang and S. A. VanSlyke, Appl. Phys. Lett. 51, 913 (1987).

${ }^{3}$ J. H. Burroughes, D. D. C. Bradley, A. R. Brown, R. N. Marks, K. Mackay, R. H. Friend, P. L. Burns, and A. B. Holmes, Nature (London) 347, 539 (1990).

${ }^{4}$ H. Koezuka, A. Tsumura, and T. Ando, Synth. Met. 18, 699 (1987).

${ }^{5}$ N. S. Sariciftci, L. Smilowitz, A. J. Heeger, and F. Wudl, Science 258, 1474 (1992).

${ }^{6}$ Charge Transport in Disordered Solids with Applications in Electronics, edited by S. Baranovski (Wiley, Chichester, UK, 2006).

${ }^{7}$ A. Miller and E. Abrahams, Phys. Rev. 120, 745 (1960).

${ }^{8}$ R. A. Marcus, J. Chem. Phys. 24, 966 (1956).

${ }^{9}$ H. Bässler, Phys. Status Solidi B 175, 15 (1993).

${ }^{10}$ P. M. Borsenberger, J. Appl. Phys. 68, 6263 (1990).

${ }^{11}$ P. M. Borsenberger, E. H. Magin, M. van der Auweraer, and F. C. de Schryver, Phys. Status Solidi A 140, 9 (1993).

${ }^{12}$ M. Gailberger and H. Bässler, Phys. Rev. B 44, 8643 (1991).

${ }^{13}$ B. Hartenstein, Chem. Phys. 191, 321 (1995).

${ }^{14}$ L. B. Schein, A. Rosenberg, and S. L. Rice, J. Appl. Phys. 60, 4287 (1986).

${ }^{15}$ M. Stolka, J. F. Yanus, and D. M. Pai, J. Phys. Chem. 88, 4707 (1984).

${ }^{16}$ G. Verbeek, Chem. Phys. Lett. 188, 85 (1992).

${ }^{17}$ M. van der Auweraer, F. C. de Schryver, and P. M. Borsenberger, Chem. Phys. 186, 409 (1994).

${ }^{18}$ K. Seki and M. Tachiya, Phys. Rev. B 65, 1 (2001).

${ }^{19}$ D. Dunlap and V. Kenkre, Chem. Phys. 178, 67 (1993).

${ }^{20}$ M. Novo, M. van der Auweraer, F. C. de Schryver, P. M. Borsenberger, and H. Bässler, Phys. Status Solidi B 177, 223 (1993).

${ }^{21}$ A. V. Vannikov, A. Y. Kryukov, A. G. Tyurin, and T. S. Zhuravleva, Phys. Status Solidi A 115, K47 (1989).

${ }^{22}$ A. Peled and L. B. Schein, Chem. Phys. Lett. 153, 422 (1988).

${ }^{23}$ R. H. Young and N. G. Rule, Phys. Rev. Lett. 72, 388 (1994).

${ }^{24}$ M. van der Auweraer, F. C. de Schryver, P. M. Borsenberger, and H. Bässler, Adv. Mater. 6, 199 (1994).

${ }^{25}$ M. Jakobsson and S. Stafström, J. Chem. Phys. 129, 125102 (2008).

${ }^{26}$ F. C. Grozema, P. T. van Duijnen, Y. A. Berlin, M. A. Ratner, and L. D. A. Siebbeles, J. Phys. Chem. B 106, 7791 (2002).

${ }^{27}$ H. C. F. Martens, P. W. M. Blom, and H. F. M. Schoo, Phys. Rev. B 61, 7489 (2000).

${ }^{28}$ S. Tretiak, A. Saxena, R. L. Martin, and A. R. Bishop, Phys. Rev. Lett. 89, 1 (2002). 